\title{
The Analysis of Corporate Bond Valuation under an Infinite Dimensional Compound Poisson Framework
}

\author{
Sheng Fan \\ School of Economic Mathematics, Southwestern University of Finance and Economics, Chengdu 611130, China \\ Correspondence should be addressed to Sheng Fan; fansheng@swufe.edu.cn
}

Received 18 March 2014; Accepted 10 May 2014; Published 28 May 2014

Academic Editor: Igor Leite Freire

Copyright (C) 2014 Sheng Fan. This is an open access article distributed under the Creative Commons Attribution License, which permits unrestricted use, distribution, and reproduction in any medium, provided the original work is properly cited.

\begin{abstract}
This paper analyzes the firm bond valuation and credit spread with an endogenous model for the pure default and callable default corporate bond. Regarding the stochastic instantaneous forward rates and the firm value as an infinite dimensional Poisson process, we provide some analytical results for the embedded American options and firm bond valuations.
\end{abstract}

\section{Introduction}

Corporate bonds trade at higher yield than the comparable government bonds due to default risk and embedded American options such as call, put, and convertibility. However, even accounts for the effect of embedded options, it has been difficult to reconcile the observed spread with credit risk and reasonable parameters and is thus frequently referred to as the "credit spread puzzle" [1]. Wide range models typically explain only $20 \%-30 \%$ of the observed credit spreads. The credit quality of bond, downward jump risk of the issuers' value, and the bond maturity have some explaining power to the observed spreads. For junk bonds, the credit risk accounts for much larger fraction of observed spreads than investment grade bonds do [2]. For long-maturity bonds, Collin-Dufresne et al. [3] have argued that firms with good credit quality are likely to issue more debt, which leads to higher yield spreads. The authors in [4-7] have shown that models with incorporating jump risk explain a significant part of observed credit spreads. Liquidity risk is an important element of spreads, if the debt market is of lower liquidity. It is shown in [8] by Longstaff et al. that liquidity risk may not construct the majority of corporate spreads as in the frictionless credit-default swaps market.

Since the structural approach is one of the most widely employed frameworks of credit risk valuation, by incorporating jump risk into the structural framework, this paper analyzes the valuation of callable corporate bond and embedded options. Allowing the economic variables driven by its own risk resource as in [9-12], we emphasize that the state nature of different maturities is an important determinant factor to the assets pricing. For the instantaneous forward interest rates, the risk resource depends not only on time $t$ but also on the time-to-maturity. Therefore, we expand the dynamics of the instantaneous forward rates as an infinite dimensional jump-diffusion process. This expansion has three features: (1) the model could flexibly capture the covariation and the term structure of correlation between different firm bonds and government bonds in a parsimonious way than their multifactor counterparts. (2) Any observed data set of bond prices is fully compatible with the expanding random process. (3) It is unnecessary to add an error term in the econometric model to calculate the parameters.

For the corporate bond valuation, based on different assumptions about the bankruptcy rules, exogenous and endogenous models have different implication and lead to different risk management behaviours. In the exogenous model, bankruptcy liquidation is made by the outside investors when the firm value reaches the trigger value such as bond par value [13-16]. For some exogenous bankruptcy models, there is arbitrage opportunity due to the negative spreads. In the endogenous model, the bankruptcy decision depends on the issuers who endogenously optimally exercise the embedded options [17-20]. We conduct our analysis within the endogenous bankruptcy framework. 
To examine the corporate bond valuation and the interaction with embedded options, we introduce a benchmark bond which is a risk-free comparable government bond as in [21]. Treating the corporate bond as the benchmark bond minus the embedded options, we could easily clarify the interaction between call provisions and default risk.

In this paper, we first analyze the impact of benchmark bond price and firm value to the embedded option as well as the corporate bond valuation. It shows that credit spread is positively related with the benchmark bond price and negatively related with firm value. For the callable corporate bond, the embedded options value is higher than that of the pure default bond. The increment can be decomposed as the "American premium" part and the "call option" part. Secondly, we consider the embedded American options exercise boundaries and show that, for the callable corporate bond, the relationship between the firm values and the boundaries is more complicated than that of pure default bond. When the firm value is lower than the call price, the exercise boundaries are higher for higher firm value. When the firm value is higher than the call price, the strike boundary decreases with the firm value. Similar results are obtained in [21]. But our results are more accurate. Finally, we emphasize the effect of the exogenous shock and firm procyclicality to the credit spread. If the exogenous shock is negative to the benchmark bond and positive to the firm value, the credit spread depends on the total effect.

The remainder of this paper is organized as follows. Section 2 presents the basic valuation framework for the corporate bond. Section 3 derives the valuation model for the pure default corporate bond and analyzes the effecting factors. Section 4 examines the impact of benchmark bond price, firm value, and their covariation to the credit spread for the default and callable corporate bond. The conclusion follows in Section 5.

\section{The Preliminary of Valuation Model}

Let $(\Omega, \Psi, P)$ be a probability space with a stochastic process $B(t, x)$ and let $N_{1}(t), \ldots, N_{M}(t)$ be independent Poisson processes, where $\Psi$ is a filtration. Assume that the instantaneous forward rates follow the dynamics

$$
d_{t} f(t, x)=\alpha(t, x) d t+\beta(t, x) d_{1} B(t, x)+\gamma(t, x) d J(t),
$$

where the stochastic process $B(t, x)$ is a generalization of the one-dimensional Brownian motion, which depends on both time $t$ and maturity $x$. Given time-to-maturity $x$, the stochastic perturbation $B(t, x)$ is a standard Brownian motion. $B(t, x)$ describes at time $t$ the different magnitude's stochastic perturbation to the forward rates with different time-to-maturity. The instantaneous forward rates constitute a continuum. Such generalization is natural and acceptable. The generalized Brownian motion exists and can be obtained by solving SPDEs as in [12]. This model is different from that of [21] in three aspects. (1) We model the instantaneous forward rates rather than the interest rates. (2) The continuous stochastic risk source is infinite dimension. (3) We incorporate the compound jump process in the model. $B(t, x)$ satisfies the following requirements.

(1) $B(t, x)$ is continuous on $t$ and $x$.

(2) $B(t, x)$ is a martingale in time $t$; that is, $E_{t}[B(t, x)]=0$, for all $x$.

(3) The variance of the increments with respect to time is equal to the time change; namely, $\operatorname{Var}\left[d_{1} B(t, x)\right]=d t$, for all $x$.

(4) The correlation of the increments, $\operatorname{corr}\left[d_{1} B(t, x)\right.$, $\left.d_{1} B(t, y)\right]=\rho(x, y)$, does not depend on time $t$.

At the same time, the correlation coefficient $\rho(x, y)$ satisfies the following requirements.

(1) Symmetric, $\rho(x, y)=\rho(y, x)$.

(2) $\rho(x, y)$ takes values between -1 and 1 .

(3) $\rho(x, x)=1$.

(4) The correlation coefficient matrix is positive semidefinite.

The notation $d_{1} B(t, x)$ means that the increment is taken with respect to the first element time $t . J(t)$ is a compound Poisson process; $J(t)=\sum_{j=1}^{N(t)} Y_{j}$. Poisson process $N(t)$ counts the number of jumps that occur at or before time $t$ with the intensity $\lambda . Y_{j}(j=1,2, \ldots)$ are independent identically distributed discrete random variables with finite values $a_{1}, a_{2}, \ldots a_{M}$; the probability $P\left(Y_{j}=a_{i}\right)=p_{i}, \sum_{i=1}^{M} p_{i}=1$. Here, we allow that the impacts of exogenous shock to the economic basis are time-variable and assume that the jump size is finite. $\alpha(t, x), \beta(t, x)$, and $\gamma(t, x)$ are nonrandom functions and satisfy Lipschitz linear growth conditions. That is, for some constant $L, \alpha, \beta$, and $\gamma$ satisfy

$$
\begin{gathered}
|\alpha(t, x)-\alpha(t, y)|+|\beta(t, x)-\beta(t, y)| \\
+|\gamma(t, x)-\gamma(t, y)| \leq L|x-y|, \\
|\alpha(t, x)|+|\beta(t, x)|+|\gamma(t, x)| \leq L(1+|x|),
\end{gathered}
$$

for all $x, y, t \in \mathfrak{R}^{+}$. In the filtration $\Psi_{t}$, the compound jump is independent of the stochastic string process $B(t, x)$.

The decomposition theorem of a compound Poisson process (see [22]) shows that $J(t)=\sum_{m=1}^{N(t)} Y_{m}$ could be rewritten as $J(t)=\sum_{i=1}^{M} a_{i} N_{i}(t)$. So the dynamics of instantaneous forward rates can be written in the integral form

$$
\begin{aligned}
f(t, x)= & f(0, x)+\int_{0}^{t} \alpha(u, x) d u+\int_{0}^{t} \beta(u, x) d_{1} B(u, x) \\
& +\sum_{i=1}^{M} \int_{0}^{t} \gamma(u, x) a_{i} d N_{i}(u) .
\end{aligned}
$$

The price at time $t$ of a zero coupon bond with maturity $s$ is expressed as

$$
P(t, s)=\exp \left[-\int_{0}^{s-t} f(t, x) d x\right] .
$$


Now, we consider a firm with one single bond to be outstanding. The bond has a series coupons paid $c_{j}$ at each time $t_{j}, 0<t_{1}<t_{2}<\cdots<t_{n}=T$, and maturity at time $T$. In order to analyze the effect of the embedded default and call options on the corporate bond, we introduce a benchmark bond which is noncallable nondefault with the same coupon and maturity as the corporate bond. Corporate bond can be viewed as the benchmark bond minus a default and/or call option. The benchmark bond with maturity $T\left(=t_{n}\right)$ can be expressed as

$$
\begin{array}{r}
P(t)=\sum_{j}^{n} c_{j} P\left(t, t_{j}\right)=\sum_{j}^{n} c_{j} \exp \left[-\int_{0}^{t_{j}-t} f(t, x) d x\right], \\
\text { for } t_{j-1} \leq t<t_{j}, \quad j=1,2, \ldots, n .
\end{array}
$$

Assume that the market is complete. By the second fundamental theorem of asset pricing (see [22]), there exists an unique risk-neutral measure $\widetilde{P}$. Under the risk-neutral measure $\widetilde{P}$, for each fixed maturity $x$, define $\widetilde{B}(t, x)=B(t, x)+$ $\int_{0}^{t} \int_{0}^{\infty} \theta(u, y) c(x, y) d y d u$, where $\theta(u, y)$ is the market price of risk. $\widetilde{B}(t, x)$ is the counterpart of $B(t, x)$ under the actual probability measure $P$. For each maturity $x, \widetilde{B}(t, x)$ is still a Brownian motion. $J(t)=\sum_{j=1}^{\widetilde{N}(t)} Y_{j}$ is a compound Poisson process with intensity $\widetilde{\lambda}$, where $\widetilde{N}(t)$ is the number of jumps till time $t$ under the risk-neutral measure $\widetilde{P}$. The jump sizes $Y_{1}, Y_{2} \ldots$ are still I.I.D. random variables, while the jump intensity satisfies $\widetilde{P}\left(Y_{j}=a_{i}\right)=\widetilde{p}\left(a_{i}\right)=\widetilde{\lambda}_{i} / \widetilde{\lambda},(i=1,2, \ldots, M)$ for arbitrary $j$, where $\sum_{i=1}^{M} \widetilde{\lambda_{i}}=\widetilde{\lambda}$ and each $\widetilde{\lambda_{i}}(i=1,2, \ldots, M)$ is a positive constant. Notice that, under the measure $\widetilde{P}$, $\widetilde{B}(t, x)$ is still independent of $\widetilde{N}_{i}(t)(i=1,2, \ldots, M)$. Under the risk-neutral measure $\widetilde{P}$, the price at time $t$ of a zero coupon bond with maturity $s$ can be expressed as (see [23])

$$
\begin{aligned}
& P(t, s) \\
& \begin{array}{r}
=P(0, s) \exp \left[\int_{0}^{t} r(u) d u\right. \\
-\int_{0}^{t} \int_{0}^{s-u} \sigma(u, x) d x d_{1} \widetilde{B}(u, x) \\
-\frac{1}{2} \int_{0}^{t} d u \int_{0}^{s-u} \sigma(u, x) \\
\times \int_{0}^{s-u} \sigma(u, y) \\
\times \rho(x, y) d y d x \\
-\sum_{i=1}^{M} \int_{0}^{t}\left[\operatorname { e x p } \left[\left(-a_{i} \int_{0}^{s-u} \gamma(u, x) d x\right)\right.\right. \\
\left.-1] \widetilde{\lambda}_{i} d u\right] \\
\times \exp \left[-\sum_{i=1}^{M} \int_{0}^{t} \int_{0}^{s-u} \gamma(u, x) d x a_{i} d \widetilde{N}_{i}(u)\right],
\end{array}
\end{aligned}
$$

where $\sigma(t, x)$ is a nonrandom function and satisfies $\widetilde{E} \int_{0}^{t} \int_{0}^{\infty}|\sigma(u, x)| \int_{0}^{\infty}|\sigma(u, y)| \rho(x, y) d y d x d u<\infty$, for all $t \geq 0 . \gamma(t, x)$ is a left-continuous nonrandom function and satisfies $\widetilde{E} \int_{0}^{t} \int_{0}^{\infty}|\gamma(u, x)| \int_{0}^{\infty}|\gamma(u, y)| \rho(x, y) d y d x d u<\infty$, for all $t \geq 0$. The discounted zero bond is a martingale. Notice that $P(s, s)=1$. We rewrite the value of zero coupon bond as

$$
\begin{aligned}
P(t, s)=\exp \{ & -\int_{t}^{s} r(u) d u \\
& +\int_{t}^{s} \int_{0}^{s-u} \sigma(u, x) d x d_{1} \widetilde{B}(u, x) \\
& +\frac{1}{2} \sigma_{f}^{2}(t, s) \\
& +\sum_{i=1}^{M} \int_{t}^{s}\left[\exp \left(-a_{i} \int_{0}^{s-u} \gamma(u, x) d x\right)-1\right] \\
& \left.+\sum_{i=1}^{M} \int_{t}^{s} \int_{0}^{s-u} \gamma(u, x) d x a_{i} d \widetilde{N}_{i}(u)\right\},
\end{aligned}
$$

where

$$
\sigma_{f}^{2}(t, s)=\int_{t}^{s} d u \int_{0}^{s-u} \sigma(u, x) \int_{0}^{s-u} \sigma(u, y) \rho(x, y) d y d x .
$$

Assume that the value of the firm $S$ is independent of its capital structure and is a jump diffusion given by

$$
\begin{aligned}
d S(t)= & (a(t)-h(t)) S(t) d t \\
& +S(t) \int_{0}^{\bar{T}} b(t, x) d_{1} B(t, x) d x \\
& +S\left(t^{-}\right) c(t) d J(t),
\end{aligned}
$$

where $\bar{T}(0<\bar{T}<\infty)$ is the survival period of the firm, $E \int_{0}^{\bar{T}} \int_{0}^{\infty}|b(t, x)| \int_{0}^{\infty}|b(t, y)| \rho(x, y) d y d x d t<\infty, a(t)$, $b(t, x)$, and $c(t)$ satisfy Lipschitz conditions, $c(t)$ is a leftcontinuous nonrandom function and satisfies, for all jump size $a_{i}, c(t)>\max _{i}\left\{-1 / a_{i}\right\}$ a.s., and $h(t) \geq 0$ is the firm's payout rate.

Under the risk-neutral measure $\widetilde{P}$, we have

$$
\begin{gathered}
d J(t)=\sum_{i=1}^{M} a_{i} d \widetilde{N}_{i}(t), \\
d_{1} B(t, x)=d_{1} \widetilde{B}(t, x)-\int_{0}^{\infty} \theta(t, y) \rho(x, y) d y d t .
\end{gathered}
$$

By the market price of risk equation

$$
\begin{aligned}
a(t)-r(t)= & \int_{0}^{\bar{T}} b(t, x) \int_{0}^{\infty} \theta(t, y) \rho(x, y) d y d x \\
& -c(t) \sum_{i=1}^{M} a_{i} \tilde{\lambda}_{i},
\end{aligned}
$$


the dynamics of the firm value is rewritten as

$$
\begin{aligned}
d S(t)= & {[r(t)-h(t)] S(t) d t } \\
& +S(t) \int_{0}^{\bar{T}} b(t, x) d_{1} \widetilde{B}(t, x) d x \\
& +S\left(t^{-}\right) \sum_{i=1}^{M} a_{i} c(t) d\left(\widetilde{N}_{i}(t)-\widetilde{\lambda}_{i} t\right),
\end{aligned}
$$

where $\widetilde{N}_{i}(t)-\widetilde{\lambda}_{i} t$ is a compensated Poisson process.

Theorem 1. Under the risk-neutral measure $\widetilde{P}$, the firm value is

$$
\begin{gathered}
S(t)=s(0) \exp \left\{\int_{0}^{t}[r(u)-h(u)\right. \\
-\frac{1}{2} \int_{0}^{\bar{T}} b(u, x) d x \\
\quad \times \int_{0}^{\bar{T}} b(u, y) \rho(x, y) d y \\
\left.\quad-\sum_{i=1}^{M} c(u) a_{i} \widetilde{\lambda_{i}}\right] d u \\
\left.+\int_{0}^{t} \int_{0}^{T} b(u, x) d \widetilde{B}_{1}(u, x) d x\right\} \\
\quad \times \exp \left\{\sum_{i=1}^{M} \int_{0}^{t} \ln \left(a_{i} c(u)+1\right) d \widetilde{N}_{i}(u)\right\} .
\end{gathered}
$$

Proof. We prove that (13) is the solution of (12). Let $S(t)=$ $S(0) \exp [X(t)]$. Denote

$$
\begin{gathered}
X^{c}(t)=\int_{0}^{t}[r(u)-h(u) \\
-\frac{1}{2} \int_{0}^{\bar{T}} b(u, x) d x \\
\quad \times \int_{0}^{\bar{T}} b(u, y) \rho(x, y) d y \\
\left.\quad-\sum_{i=1}^{M} c(u) a_{i} \widetilde{\lambda}_{i}\right] d u \\
+\int_{0}^{t} \int_{0}^{\bar{T}} b(u, x) d d_{1} \widetilde{B}(u, x) d x \\
X^{J}(t)=\sum_{i=1}^{M} \int_{0}^{t} \ln \left(a_{i} c(u)+1\right) d \widetilde{N}_{i}(u) .
\end{gathered}
$$

By Itô formula for jump process, we have

$$
\begin{aligned}
d S(t)= & S(t) d X^{c}(t)+\frac{1}{2} S(t) d X^{c}(t) d X^{c}(t) \\
& +S(t)-S\left(t^{-}\right) \\
= & S(t)\left\{\left[r(t)-h(t)-\sum_{i=1}^{M} c(t) a_{i} \widetilde{\lambda}_{i}\right.\right. \\
& \left.\quad-\frac{1}{2} \int_{0}^{\bar{T}} b(t, x) d x \int_{0}^{\bar{T}} b(t, y) \rho(x, y) d y\right] d t \\
& \left.+\int_{0}^{\bar{T}} b(t, x) d_{1} \widetilde{B}(t, x) d x\right\} \\
& +\frac{1}{2} S(t) \int_{0}^{\bar{T}} b(t, x) d x \int_{0}^{\bar{T}} b(t, y) \rho(x, y) d y d t \\
& +S\left(t^{-}\right)\left\{\exp \left[\sum_{i=1}^{M} \ln \left(a_{i} c(t)+1\right) d \widetilde{N}_{i}(t)\right]-1\right\} .
\end{aligned}
$$

Rearranging it, we obtain

$$
\begin{aligned}
d S(t)= & S(t)\left\{\left[r(t)-h(t)-\sum_{i=1}^{M} c(t) a_{i} \widetilde{\lambda}_{i}\right] d t\right. \\
& \left.+\int_{0}^{\bar{T}} b(t, x) d_{1} \widetilde{B}(t, x) d x\right\} \\
& +S\left(t^{-}\right) \sum_{i=1}^{M} c(t) a_{i} d \widetilde{N}_{i}(t) .
\end{aligned}
$$

It completes the proof.

For $t \leq \tau \leq \bar{T}$, the value of corporate bond can be rewritten as

$$
\begin{aligned}
& S(\tau)=s(t) \exp \left\{\int_{t}^{\tau}\left[r(u)-h(u)-\sum_{i=1}^{M} c(u) a_{i}{\widetilde{\lambda_{i}}}_{i}\right] d u\right. \\
&-\frac{1}{2} \sigma_{S}^{2}(t, \tau)+\int_{t}^{\tau} \int_{0}^{\bar{T}} b(u, x) d_{1} \widetilde{B}(u, x) d x \\
&\left.+\sum_{i=1}^{M} \int_{t}^{\tau} \ln \left(a_{i} c(u)+1\right) d \widetilde{N}_{i}(u)\right\},
\end{aligned}
$$

where

$$
\sigma_{S}^{2}(t, \tau)=\int_{t}^{\tau} d u \int_{0}^{\bar{T}} b(u, x) d x \int_{0}^{\bar{T}} b(u, y) \rho(x, y) d y .
$$

\section{Valuing Defaultable Bond}

In the endogenous bankruptcy model, the bankruptcy decision is optimally done by the bond issuers' themselves. For the 
pure default noncallable bond, when the embedded default option arrives at its maximum, the issuer would give up the firm to exempt its debt. The value of the pure defaultable bond with embedded default option is

$$
P_{D}(t)=P(t)-f_{D}(t),
$$

where $f_{D}(t)$ is the embedded default option value at time $t$. Consider

$$
f_{D}(t) \equiv \sup _{t \leq \tau \leq T} \widetilde{E}\left\{e^{-\int_{t}^{\tau} r(u) d u}[P(\tau)-S(\tau)]^{+} \mid \Psi_{t}\right\},
$$

where $\Psi_{t}, 0 \leq t \leq T$ is the filtration; $f_{D}(t)$ satisfies

$$
f_{D}(t) \geq(P(t)-S(t))^{+} .
$$

The yield spread of a given bond over its benchmark bond is a straightforward transformation of the bond's embedded option value. Define

$$
\tau^{*}=\inf \left\{t \geq 0: f_{D}(t)=(P(t)-S(t))^{+}\right\},
$$

as the optimal stopping time based on the state $S(t)=s^{*}$ at time $t$, which is the first time the default option value $f_{D}(t)$ reaches the level $(P(t)-S(t))^{+}$.

Theorem 2. For different initial firm values and benchmark bond prices, the following properties hold.

(1) If $S^{x}(0)>S^{y}(0)$, then

$$
-\tilde{p}^{s}\left[S^{x}(t)-S^{y}(t)\right] \leq f_{D}^{x}(t)-f_{D}^{y}(t) \leq 0 .
$$

(2) If $S^{x}(0)>S^{y}(0)$, then $P_{D}^{x}(t) \leq P_{D}^{y}(t)$.

(3) If $P^{x}(0)>P^{y}(0)$, then

$$
\begin{aligned}
& 0 \leq f_{D}^{x}(t)-f_{D}^{y}(t) \leq \tilde{p}^{p}\left[P^{x}(t)-P^{y}(t)\right] . \\
& \text { (4) If } P^{x}(0)>P^{y}(0) \text {, then } P_{D}^{x}(t) \geq P_{D}^{y}(t) \text {. }
\end{aligned}
$$

Here $\widetilde{p}^{s}=\widetilde{P}\left\{P\left(\tau^{y}\right)>S^{y}\left(\tau^{y}\right) \mid S(t)=s^{y}\right\}$ is the risk-neutral default probability under the condition that the firm value at time $t$ is $S(t)=s$ and $\widetilde{p}^{p}=\widetilde{P}\left\{P^{x}\left(\tau^{x}\right)>S\left(\tau^{x}\right) \mid P(t)=p^{x}\right\}$ is the risk-neutral default probability under the state in which the benchmark bond price at time $t$ is $P(t)=p$.

Remark 3. These results are consistent with those of [21], but our results are more accurate by providing closer embedded options spread.

Proof. Due to $c(t) a_{i}>-1, i=1,2, \ldots, M$, by the no-crossing properties for jump-diffusion process, we have

$$
\begin{aligned}
& S^{x}(0)>S^{y}(0) \Longrightarrow S^{x}(t) \geq S^{y}(t), \\
& \widetilde{P}-\text { a.s. } \quad \forall 0<t<\infty .
\end{aligned}
$$

(1) Given that the state at time $t$ is $P(t)=p$ and $S(t)=s^{x}$, assume that its optimal stopping time is $\tau^{x}$ and its associated embedded default options value is $f_{D}^{x}(t)$. Thus
$f_{D}^{x}(t)=\widetilde{E}\left\{e^{-\int_{t}^{\tau^{x}} r(u) d u}\left[P\left(\tau^{x}\right)-S^{x}\left(\tau^{x}\right)\right]^{+} \mid \Psi_{t}\right\}$. Similarly, $f_{D}^{y}(t)$ is the embedded options value when $S(t)=s^{y}$ at time $t$. Thus

$$
\begin{aligned}
f_{D}^{y}(t)- & f_{D}^{x}(t) \\
= & \sup _{t \leq \tau \leq T} \widetilde{E}\left\{e^{-\int_{t}^{\tau} r(u) d u}\left[P(\tau)-S^{y}(\tau)\right]^{+} \mid \Psi_{t}\right\} \\
& -\widetilde{E}\left\{e^{-\int_{t}^{\tau^{x}} r(u) d u}\left[P\left(\tau^{x}\right)-S^{x}\left(\tau^{x}\right)\right]^{+} \mid \Psi_{t}\right\} \\
\geq \widetilde{E} & \left\{e^{-\int_{t}^{\tau^{x}} r(u) d u}\left[P\left(\tau^{x}\right)-S^{y}\left(\tau^{x}\right)\right]^{+}\right. \\
& \left.\quad-e^{-\int_{t}^{\tau^{x}} r(u) d u}\left[P\left(\tau^{x}\right)-S^{x}\left(\tau^{x}\right)\right]^{+} \mid \Psi_{t}\right\}
\end{aligned}
$$

$\geq 0$.

For $S(t)=s^{y}$, assume that its optimal stopping time is $\tau^{y}$. We have

$$
\begin{aligned}
& f_{D}^{y}(t)-f_{D}^{x}(t) \\
& \leq \widetilde{E}\left\{e^{-\int_{t}^{\tau^{y}} r(u) d u}\left(P\left(\tau^{y}\right)-S^{y}\left(\tau^{y}\right)\right)^{+}\right. \\
& \left.-e^{-\int_{t}^{\tau^{y}} r(u) d u}\left(P\left(\tau^{y}\right)-S^{x}\left(\tau^{y}\right)\right)^{+} \mid \Psi_{t}\right\} \\
& =\widetilde{E}\left\{\left[e^{-\int_{t}^{\tau^{y}} r(u) d u}\left(P\left(\tau^{y}\right)-S^{y}\left(\tau^{y}\right)\right)\right.\right. \\
& \left.-e^{-\int_{t}^{\tau^{y}} r(u) d u}\left(P\left(\tau^{y}\right)-S^{x}\left(\tau^{y}\right)\right)^{+}\right] \\
& \left.\times I_{\left\{P\left(\tau^{y}\right)>S^{y}\left(\tau^{y}\right)\right\}} \mid \Psi_{t}\right\} \\
& \leq \widetilde{E}\left\{\left[e^{-\int_{t}^{\tau^{y}} r(u) d u}\left(P\left(\tau^{y}\right)-S^{y}\left(\tau^{y}\right)\right)\right.\right. \\
& \left.-e^{-\int_{t}^{\tau^{y}} r(u) d u}\left(P\left(\tau^{y}\right)-S^{x}\left(\tau^{y}\right)\right)^{+}\right] \\
& \left.\times I_{\left\{P\left(\tau^{y}\right)>S^{y}\left(\tau^{y}\right)\right\}} \mid \Psi_{t}\right\} \\
& =\widetilde{E}\left\{e^{-\int_{t}^{\tau^{y}} r(u) d u}\left[S^{x}\left(\tau^{y}\right)-S^{y}\left(\tau^{y}\right)\right] \mid \Psi_{t}\right\} \widetilde{P} \\
& \times\left\{P\left(\tau^{y}\right)>S^{y}\left(\tau^{y}\right)\right\} \\
& =\left[S^{x}(t)-S^{y}(t)\right] \widetilde{p}^{s},
\end{aligned}
$$

where $I_{A}$ denotes the indicator function of the set $A$. In the second last equation, we use the fact that $\widetilde{E}\left\{X I_{A} \mid \Psi_{t}\right\}=\widetilde{E}(X \mid$ $\left.\Psi_{t}\right) \widetilde{P}(A)$ (by the same token, (30), (40), and (47) also hold).

(2) Note that $P_{D}(t)=P(t)-f_{D}(t)$. For firm value $S^{x}(t)>$ $S^{y}(t)$, we have

$$
P_{D}^{x}(t)=P(t)-f_{D}^{x}(t) \leq P(t)-f_{D}^{y}(t)=P_{D}^{y}(t) .
$$


(3) By the no-crossing properties for the jump-diffusion process, for $P^{x}(0)>P^{y}(0)$, we have

$$
\begin{aligned}
& P^{x}(0)>P^{y}(0) \\
& \Longrightarrow r^{x}(0) \leq r^{y}(0) \\
& \Longrightarrow r^{x}(t) \leq r^{y}(t) \\
& \Longrightarrow e^{-\int_{0}^{t} r^{x}(u) d u} \geq e^{-\int_{0}^{t} r^{y}(u) d u}, \\
& \widetilde{P}-\text { a.s. } \quad \forall 0<t<\infty, \\
& P^{x}(0)>P^{y}(0) \Longrightarrow P^{x}(t) \geq P^{y}(t), \\
& \widetilde{P}-\text { a.s. } \quad \forall 0<t<\infty .
\end{aligned}
$$

Given that the state at time $t$ is $P(t)=p^{y}$ with the optimal stopping time $\tau^{y}$, we have

$$
\begin{aligned}
& f_{D}^{x}(t)-f_{D}^{y}(t) \\
& \geq \widetilde{E}\left\{e^{-\int_{t}^{\tau^{y}} r^{x}(u) d u}\left[P^{x}\left(\tau^{y}\right)-S\left(\tau^{y}\right)\right]^{+} \mid \Psi_{t}\right\} \\
& -\widetilde{E}\left\{e^{-\int_{t}^{\tau^{y}} r^{y}(u) d u}\left[P^{y}\left(\tau^{y}\right)-S\left(\tau^{y}\right)\right]^{+} \mid \Psi_{t}\right\} \\
& =\widetilde{E}\left\{e^{-\int_{t}^{\tau^{y}} r^{x}(u) d u}\left[P^{x}\left(\tau^{y}\right)-S\left(\tau^{y}\right)\right]^{+}\right. \\
& \left.-e^{-\int_{t}^{\tau^{y}} r^{y}(u) d u}\left[P^{y}\left(\tau^{y}\right)-S\left(\tau^{y}\right)\right]^{+} \mid \Psi_{t}\right\}
\end{aligned}
$$

$\geq 0$.

For the state of the value $P(t)=p^{x}$ with the optimal stopping time $\tau^{x}$, we have

$$
\begin{aligned}
& f_{D}^{x}(t)- f_{D}^{y}(t) \\
& \leq \widetilde{E}\left\{e^{-\int_{t}^{\tau^{x}} r^{x}(u) d u}\left[P^{x}\left(\tau^{x}\right)-S\left(\tau^{x}\right)\right]^{+}\right. \\
&\left.-e^{-\int_{t}^{\tau^{x}} r^{y}(u) d u}\left[P^{y}\left(\tau^{x}\right)-S\left(\tau^{x}\right)\right]^{+} \mid \Psi_{t}\right\} \\
&=\widetilde{E}\left\{\left[e^{-\int_{t}^{\tau^{x}} r^{x}(u) d u}\left[P^{x}\left(\tau^{x}\right)-S\left(\tau^{x}\right)\right]\right.\right. \\
&\left.-e^{-\int_{t}^{\tau^{x}} r^{y}(u) d u}\left(P^{y}\left(\tau^{x}\right)-S\left(\tau^{x}\right)\right)^{+}\right] \\
&\left.\cdot I_{\left\{P^{x}\left(\tau^{x}\right)>S\left(\tau^{x}\right)\right\}} \mid \Psi_{t}\right\} \\
& \widetilde{E}\left\{\left[e^{-\int_{t}^{\tau^{x}} r^{x}(u) d u}\left[P^{x}\left(\tau^{x}\right)-S\left(\tau^{x}\right)\right]\right.\right. \\
&\left.-e^{-\int_{t}^{\tau^{x}} r^{y}(u) d u}\left(P^{y}\left(\tau^{x}\right)-S\left(\tau^{x}\right)\right)\right]
\end{aligned}
$$

$$
\begin{aligned}
&\left.I_{\left\{P^{x}\left(\tau^{x}\right)>S\left(\tau^{x}\right)\right\}} \mid \Psi_{t}\right\} \\
& \leq \widetilde{E}\left\{e^{-\int_{t}^{\tau^{x}} r^{x}(u) d u}\left[P^{x}\left(\tau^{x}\right)-S\left(\tau^{x}\right)\right]\right. \\
&\left.-e^{-\int_{t}^{\tau^{x}} r^{y}(u) d u}\left[P^{y}\left(\tau^{x}\right)-S\left(\tau^{x}\right)\right] \mid \Psi_{t}\right\} \\
& \times \widetilde{P}\left\{P^{x}\left(\tau^{x}\right)>S\left(\tau^{x}\right)\right\} \\
& \leq \widetilde{E}\left\{e^{-\int_{t}^{\tau^{x}} r^{x}(u) d u} P^{x}\left(\tau^{x}\right)\right. \\
&\left.-e^{-\int_{t}^{\tau^{x}} r^{y}(u) d u} P^{y}\left(\tau^{x}\right) \mid \Psi_{t}\right\} \widetilde{p}^{p} \\
&=\left[P^{x}(t)-P^{y}(t)\right] \widetilde{p}^{p} .
\end{aligned}
$$

(4) Note that $P_{D}(t)=P(t)-f_{D}(t)$. For $P^{x}(t) \geq P^{y}(t)$, we have

$$
P_{D}^{x}(t)=P^{x}(t)-f_{D}^{x}(t) \geq P^{y}(t)-f_{D}^{y}(t)=P_{D}^{y}(t) .
$$

This completes the proof.

\section{Valuing Callable Defaultable Bond}

For the callable defaultable bond, when the firm value falls far below its obligation such that the default option arrives at its maximum, the issuers would exempt its obligation by giving up the firm. When the benchmark bond exceeds the preset provisional call price schedule $K_{t}$, which is the strike price to call the debt, the issuers intend to exercise the call option to buy back the bond or reissue a bond with lower interest rates. The value of the callable corporate bond is

$$
P_{C D}(t)=P(t)-f_{C D}(t)
$$

where $f_{C D}(t)$ is the embedded option value at time $t$,

$$
\begin{aligned}
& f_{C D}(t) \\
& \equiv \sup _{t \leq \tau \leq T} \widetilde{E}\left\{\exp \left[-\int_{t}^{\tau} r(u) d u\right]\left[P(\tau)-K_{\tau} \wedge S(\tau)\right]^{+} \mid \Psi_{t}\right\} .
\end{aligned}
$$

The embedded option $f_{C D}(t)$ satisfies

$$
f_{C D}(t) \geq\left(P(t)-K_{t} \wedge S(t)\right)^{+}
$$

The optimal stopping time is

$$
\tau=\inf \left\{t \geq 0: f_{C D}(t)=\left(P(t)-K_{t} \wedge S(t)\right)^{+}\right\}
$$

Similarly, we have the following theorem. 
Theorem 4. For the different initial value of firm value and benchmark bond, the following properties hold.

(1) If $S^{x}(0)>S^{y}(0)$, then

$$
-\left[S^{x}(t)-S^{y}(t)\right] \widetilde{p}^{s} \leq f_{C D}^{x}(t)-f_{C D}^{y}(t) \leq 0 .
$$

(2) If $S^{x}(0)>S^{y}(0)$, then $P_{C D}^{x} \leq P_{C D}^{y}(t)$.

(3) If $P^{x}(0)>P^{y}(0)$, then

$$
-\left[S^{x}(t)-S^{y}(t)\right] \tilde{p}^{p} \leq f_{C D}^{x}(t)-f_{C D}^{y}(t) \leq 0 .
$$

(4) If $P^{x}(0)>P^{y}(0)$, then $P_{C D}^{x} \geq P_{C D}^{y}(t)$.

Proof. It is similar with the case of the pure default corporate bond. By the no-crossing properties for jump-diffusion process, we have

$$
\begin{aligned}
& S^{x}(0)>S^{y}(0) \\
& \Longrightarrow S^{x}(t) \geq S^{y}(t) \\
& \Longrightarrow K_{t} \wedge S^{x}(t) \geq K_{t} \wedge S^{y}(t), \\
& \widetilde{P} \text { - a.s. } \quad \forall 0<t<\infty .
\end{aligned}
$$

So, replacing the strike price $S^{x}(t)$ by $K_{t} \wedge S^{x}(t)$ and $S^{y}(t)$ by $K_{t} \wedge S^{y}(t)$ in the proof of Theorem 2 , we obtain the results of Theorem 4 .

Theorem 5. $f_{C D}(t)$ satisfies

$$
\begin{aligned}
& \text { (1) } f_{C D}(t)-f_{D}(t) \\
& \quad=A P(t)+\widetilde{E}\left\{e^{-\int_{t}^{\tau_{d}} r(u) d u}\left[P\left(\tau_{d}\right) \wedge S\left(\tau_{d}\right)-K_{\tau_{d}}\right]^{+} \mid \Psi_{t}\right\}
\end{aligned}
$$

(2) $f_{C D}(t)-f_{D}(t) \leq f_{C}(t)$,

where

$$
\begin{aligned}
& A P(t)=\widetilde{E}\left\{e^{-\int_{t}^{\tau_{c d}} r(u) d u}\left[P\left(\tau_{c d}\right)-K_{\tau_{c d}} \wedge S\left(\tau_{c d}\right)\right]^{+}\right. \\
& \left.-e^{-\int_{t}^{\tau_{d}} r(u) d u}\left[P\left(\tau_{d}\right)-K_{\tau_{d}} \wedge S\left(\tau_{d}\right)\right]^{+} \mid \Psi_{t}\right\}, \\
& f_{C}(t)=\sup _{t \leq \tau \leq T} \widetilde{E}\left\{e^{-\int_{t}^{\tau} r(u) d u}\left[P(\tau)-K_{\tau}\right]^{+} \mid \Psi_{t}\right\} \text {, }
\end{aligned}
$$

where $\tau_{d}$ is the optimal stopping time for the pure default corporate bond and $\tau_{c d}$ is the optimal stopping time for the callable default corporate bond.

Remark 6. Due to the embedded call option, $f_{C D}(t)$ is worth more than $f_{D}(t)$. The increment of $f_{C D}(t)$ over $f_{D}(t)$ can be decomposed into two parts.

(1) The part of the "American premium" $(\mathrm{AP}(t))$. That is, the firm has the right to exercise the embedded option at the optimal stopping time.
(2) The part of the "call option" $\widetilde{E}\left\{\exp \left[-\int_{t}^{\tau_{d}} r(u) d u\right]\left[P\left(\tau_{d}\right) \wedge S\left(\tau_{d}\right)-K_{\tau_{d}}\right]^{+} \quad \mid \quad \Psi_{t}\right\}$. Due to the call provision, the firm has the option either to buy back the debt when the firm works well $\left(K_{\tau_{d}} \leq P\left(\tau_{d}\right)<S\left(\tau_{d}\right)\right)$ or to keep the firm when the firm value is less than the benchmark bond but before bankruptcy $\left(K_{\tau_{d}} \leq S\left(\tau_{d}\right) \leq P\left(\tau_{d}\right)\right)$.

From the second part of Theorem 4, the increment of $f_{C D}(t)$ over $f_{D}(t)$ is less than that of embedded pure call option $f_{C}(t)$ due to the interaction between the default option and call option.

Proof. (1) Notice that $S(t) \geq K_{t} \wedge S(t)$. Let $\tau_{c d}$ be the optimal stopping time of $f_{C D}(t)$, and let $\tau_{d}$ be the optimal stopping time of $f_{D}(t)$,

$$
\begin{aligned}
& f_{C D}(t)- f_{D}(t) \\
&= \widetilde{E}\left\{e^{-\int_{t}^{\tau_{c d}} r(u) d u}\left[P\left(\tau_{c d}\right)-K_{\tau_{c d}} \wedge S\left(\tau_{c d}\right)\right]^{+} \mid \Psi_{t}\right\} \\
&-\widetilde{E}\left\{e^{-\int_{t}^{\tau_{d}} r(u) d u}\left[P\left(\tau_{d}\right)-S\left(\tau_{d}\right)\right]^{+} \mid \Psi_{t}\right\} \\
&= \widetilde{E}\left\{e^{-\int_{t}^{\tau_{c} d} r(u) d u}\left[P\left(\tau_{c d}\right)-K_{\tau_{c d}} \wedge S\left(\tau_{c d}\right)\right]^{+}\right. \\
&\left.\quad-e^{-\int_{t}^{\tau_{d}} r(u) d u}\left[P\left(\tau_{d}\right)-K_{\tau_{d}} \wedge S\left(\tau_{d}\right)\right]^{+} \mid \Psi_{t}\right\} \\
&+ \widetilde{E}\left\{e^{-\int_{t}^{\tau_{d}} r(u) d u}\left(P\left(\tau_{d}\right)-K_{\tau_{d}}\right)^{+} \mid \Psi_{t}\right\} \widetilde{P} \\
& \times\left[K_{\tau_{d}} \leq P\left(\tau_{d}\right)<S\left(\tau_{d}\right)\right] \\
&+ \widetilde{E}\left\{e^{-\int_{t}^{\tau_{d}} r(u) d u}\left(S\left(\tau_{d}\right)-K_{\tau_{d}}\right)^{+} \mid \Psi_{t}\right\} \\
& 1 \times \widetilde{P}\left[K_{\tau_{d}} \leq S\left(\tau_{d}\right) \leq P\left(\tau_{d}\right)\right] \\
&= \mathrm{AP}(t)+\widetilde{E}\left\{e^{-\int_{t}^{\tau_{d}} r(u) d u}\right. \\
&\left.\quad \times\left[P\left(\tau_{d}\right) \wedge S\left(\tau_{d}\right)-K_{\tau_{d}}\right]^{+} \mid \Psi_{t}\right\} .
\end{aligned}
$$

(2) On the other hand, we have

$$
\begin{aligned}
f_{C D}(t) & -f_{D}(t) \\
= & \sup _{t \leq \tau \leq T} \widetilde{E}\left\{e^{-\int_{t}^{\tau} r(u) d u}\left[P(\tau)-K_{\tau} \wedge S(\tau)\right]^{+} \mid \Psi_{t}\right\} \\
& \quad-\sup _{t \leq \tau \leq T} \widetilde{E}\left\{e^{-\int_{t}^{\tau} r(u) d u}[P(\tau)-S(\tau)]^{+} \mid \Psi_{t}\right\} \\
\leq & \sup _{t \leq \tau \leq T} \widetilde{E}\left\{e^{-\int_{t}^{\tau} r(u) d u}\left[P(\tau)-K_{\tau}\right]^{+}\right. \\
& \left.\quad+[P(\tau)-S(\tau)]^{+} \mid \Psi_{t}\right\} \\
& \quad-\sup _{t \leq \tau \leq T} \widetilde{E}\left\{e^{-\int_{t}^{\tau} r(u) d u}[P(\tau)-S(\tau)]^{+} \mid \Psi_{t}\right\} \\
\leq & \sup _{t \leq \tau \leq T} \widetilde{E}\left\{e^{-\int_{t}^{\tau} r(u) d u}\left[P(\tau)-K_{\tau}\right]^{+} \mid \Psi_{t}\right\} .
\end{aligned}
$$

This completes the proof. 
For the embedded options exercise boundaries, denote $P_{D B}^{*}$ as the exercise boundary of the pure defaultable corporate bond, when $S(t)=s^{*}$ at time $t$, which means that as soon as the benchmark bond price reaches $P_{D B}^{*}$, the firm should exercise options; that is, the firm should default. Denote $P_{C D B}^{*}$ as the exercise boundary of the callable defaultable corporate bond, when $S(t)=s^{*}$ at time $t$, which means that, in the case $K_{t}>S(t)$, the firm would default as soon as the benchmark bond price reaches $P_{C D B}^{*}$; in the case $K_{t} \leq S(t)$, the firm would call back its debt as soon as the benchmark bond price reaches $P_{C D B}^{*}$. In terms of the benchmark bond price, we have the following theorem.

Theorem 7. For each $t \in[0, T)$, it holds that

(1) if $S^{x}(t)>S^{y}(t)$, then $P_{D B}^{x}(t) \geq P_{D B}^{y}(t)$,

(2) when $K_{t}>S(t)$, if $S^{x}(t)>S^{y}(t)$, then

$$
P_{C D B}^{x}(t) \geq P_{C D B}^{y}(t),
$$

(3) when $K_{t} \leq S(t)$, if $S^{x}(t)>S^{y}(t)$, then

$$
P_{C D B}^{x}(t) \leq P_{C D B}^{y}(t),
$$

(4) if $S(t) \leq K_{t}$, then $P_{C D B}(t) \geq P_{D B}(t)$.

Proof. (1) When the benchmark bond price $P(t)<P_{D B}^{y}(t)$, the firm with $S^{y}(t)$ would not exercise the embedded default option. By part 1 of Theorem 2, we have

$$
\begin{aligned}
f_{D}^{x}(t) & \geq f_{D}^{y}(t)-\left(S^{x}(t)-S^{y}(t)\right) \\
& >P(t)-S^{y}(t)-\left(S^{x}(t)-S^{y}(t)\right) \\
& =P(t)-S^{x}(t) .
\end{aligned}
$$

So the firm with $S^{x}(t)$ would also not exercise the embedded default option, which means that the default boundary is no lower than that of the firm value $S^{y}(t)$. Thus, $P_{D B}^{x}(t) \geq P_{D B}^{y}(t)$.

(2) When $K_{t}>S(t)$, the firm would consider to exercise the default option. If $S^{x}(t)>S^{y}(t)$, for $P(t)<P_{C D B}^{y}(t)$, we have

$$
\begin{aligned}
f_{C D}^{x}(t) & \geq f_{C D}^{y}(t)-\left(S^{x}(t)-S^{y}(t)\right) \\
& \geq P(t)-S^{y}(t)-\left(S^{x}(t)-S^{y}(t)\right) \\
& =P(t)-S^{x}(t) .
\end{aligned}
$$

So $P_{C D B}^{x}(t) \geq P_{C D B}^{y}(t)$.

(3) When $K_{t} \leq S(t)$, the firm would consider to exercise the call option. If $S^{x}(t)>S^{y}(t)$, which means $P(t)-K_{t} \wedge$ $S^{y}(t) \geq P(t)-K_{t} \wedge S^{x}(t)$, for $P(t)<P_{C D B}^{x}(t)$, we have

$$
f_{C D}^{y}(t) \geq f_{C D}^{x}(t)>\left(P(t)-K_{t}\right)^{+} .
$$

So $P(t)<P_{C D B}^{y}(t)$, which means $P_{C D B}^{x}(t) \leq P_{C D B}^{y}(t)$.

(4) When $S(t) \leq K_{t}$, for $P(t)<P_{D B}(t)$, we have

$$
f_{C D}(t) \geq f_{D}(t) \geq(P(t)-S(t))^{+}>P(t)-K_{t} \wedge S(t) .
$$

So $P(t)<P_{C D B}(t)$, which means $P_{C D B}(t) \geq P_{D B}(t)$. This completes the proof.
For the value of the embedded option, it can be reexpressed as

$$
\begin{aligned}
& f_{C D}(t) \\
& =\sup _{t \leq \tau \leq T} \widetilde{E}\left\{e^{-\int_{t}^{\tau} r(u) d u}\left[P(\tau)-K_{\tau} \wedge S(\tau)\right]^{+}\right. \\
& \left.\times I_{P(\tau)>K_{\tau} \wedge S(\tau)} \mid \Psi_{t}\right\} \\
& =\sup _{t \leq \tau \leq T} \widetilde{E}\left\{e ^ { - \int _ { t } ^ { \tau } r ( u ) d u } \left[\left(P(\tau)-K_{\tau}\right)^{+} \mid K_{\tau}\right.\right. \\
& \left.<S(\tau), \Psi_{t}\right] \times \widetilde{P}\left(K_{\tau}<S(\tau)\right) \\
& +e^{-\int_{t}^{\tau} r(u) d u}\left[(P(\tau)-S(\tau))^{+} \mid K_{\tau} \geq S(\tau), \Psi_{t}\right] \\
& \left.\times \widetilde{P}\left(K_{\tau} \geq S(\tau)\right)\right\} \\
& =\sup _{t \leq \tau \leq T} \widetilde{E}\left\{e^{-\int_{t}^{\tau} r(u) d u}\right. \\
& \times\left[\left(P(\tau)-K_{\tau}\right) \mid K_{\tau}<S(\tau),\right. \\
& \left.K_{\tau}<P(\tau), \Psi_{t}\right] \widetilde{P}\left(K_{\tau} \geq S(\tau)\right) \\
& \times \widetilde{P}\left(K_{\tau}<S(\tau)\right) \widetilde{P} \\
& +e^{-\int_{t}^{\tau} r(u) d u}\left[(P(\tau)-S(\tau)) \mid K_{\tau} \geq S(\tau),\right. \\
& \left.P(\tau) \geq S(\tau), \Psi_{t}\right] \widetilde{P}\left(K_{\tau} \geq S(\tau)\right) \\
& \times \widetilde{P}(P(\tau) \geq S(\tau))\} .
\end{aligned}
$$

For $K_{\tau}<S(\tau)$, we have

$$
\begin{aligned}
& \widetilde{P}\left\{\ln K_{\tau}<\ln S(t)+\int_{t}^{\tau}[r(u)-h(u)] d u\right. \\
& \left.+I_{S}(t, \tau)-\frac{1}{2} \sigma_{S}^{2}(t, \tau)+J_{S}(t, \tau)-C_{J S}(t, \tau)\right\} \\
& =\widetilde{P}\left\{\ln K_{\tau}-\ln S(t)-\int_{t}^{\tau}[r(u)-h(u)] d u\right. \\
& \left.<I_{S}(t, \tau)-\frac{1}{2} \sigma_{S}^{2}(t, \tau)+J_{S}(t, \tau)-C_{J S}(t, \tau)\right\},
\end{aligned}
$$

where

$$
\begin{gathered}
I_{S}(t, \tau)=\int_{t}^{\tau} \int_{0}^{\bar{T}} b(u, x) d x d_{1} \widetilde{B}(u, x), \\
J_{S}(t, \tau)=\sum_{i=1}^{M} \int_{t}^{\tau} \ln \left(a_{i} c(u)+1\right) d \widetilde{N}_{i}(u) \\
C_{J S}(t, \tau)=\sum_{i=1}^{M} \int_{t}^{\tau} c(u) a_{i} \widetilde{\lambda_{i}} d u .
\end{gathered}
$$


Notice that the price of benchmark bond satisfies

$$
P(t, \tau) P(\tau)=P(t)-\sum_{t \leq t_{j}<\tau} c_{j} P\left(t, t_{j}\right) .
$$

For $\widetilde{P}\left(K_{\tau}<P(\tau)\right)$, we have

$$
\begin{aligned}
& \widetilde{P}\left\{K_{\tau} P(t, \tau)<P(t)-\sum_{t \leq t_{j}<\tau} c_{j} P\left(t, t_{j}\right)\right\} \\
& =\widetilde{P}\left\{\ln K_{\tau}-\int_{t}^{\tau} r(u) d u+I_{f}(t, \tau)\right. \\
& +\frac{1}{2} \sigma_{f}^{2}(t, \tau)+J_{f}(t, \tau)+C_{J f}(t, \tau) \\
& \left.<\ln \left[p(t)-\sum_{t \leq t_{j}<\tau} c_{j} P\left(t, t_{j}\right)\right]\right\} \\
& =\widetilde{P}\left\{I_{f}(t, \tau)+\frac{1}{2} \sigma_{f}^{2}(t, \tau)+J_{f}(t, \tau)+C_{J f}(t, \tau)\right. \\
& <\int_{t}^{\tau} r(u) d u-\ln K_{\tau} \\
& \left.+\ln \left[p(t)-\sum_{t \leq t_{j}<\tau} c_{j} P\left(t, t_{j}\right)\right]\right\},
\end{aligned}
$$

where

$$
\begin{gathered}
I_{f}(t, \tau)=\int_{t}^{\tau} \int_{0}^{\tau-u} \sigma(u, x) d x d_{1} \widetilde{B}(u, x), \\
J_{f}(t, \tau)=\sum_{i=1}^{M} \int_{t}^{\tau} \int_{0}^{\tau-u} \gamma(u, x) d x a_{i} d \widetilde{N}_{i}(u), \\
C_{J f}(t, \tau)=\sum_{i=1}^{M} \int_{t}^{\tau}\left[\exp \left(-a_{i} \int_{0}^{\tau-u} \gamma(u, x) d x\right)-1\right] \widetilde{\lambda_{i}} d u .
\end{gathered}
$$

For $\widetilde{P}(P(\tau) \geq S(\tau))$, we have

$$
\begin{aligned}
& \widetilde{P}\{P(\tau) \geq S(\tau)\} \\
& =\widetilde{P}\left\{P(t)-\sum_{t \leq t_{j}<\tau} c_{j} P\left(t, t_{j}\right)\right. \\
& \quad \geq S(t) \exp \left[I_{f}(t, \tau)+\frac{1}{2} \sigma_{f}^{2}(t, \tau)+J_{f}(t, \tau)\right. \\
& \quad+C_{J f}(t, \tau)-\int_{t}^{\tau} h(u) d u+I_{S}(t, \tau) \\
& \left.\left.\quad-\frac{1}{2} \sigma_{S}^{2}(t, \tau)+J_{S}(t, \tau)-C_{J S}(t, \tau)\right]\right\}
\end{aligned}
$$

$$
\begin{aligned}
=\widetilde{P}\{ & I_{S f}(t, \tau)+\sigma_{f}^{2}(t, \tau)-\frac{1}{2} \sigma_{S f}^{2}(t, \tau)+\operatorname{cov}\left(I_{f}, I_{S}\right) \\
& +J_{f}(t, \tau)+C_{J f}(t, \tau)+J_{S}(t, \tau)-C_{J S}(t, \tau) \\
& \leq \ln \left[P(t)-\sum_{t \leq t_{j}<\tau} c_{j} P\left(t, t_{j}\right)\right] \\
& \left.+\int_{t}^{\tau} h(u) d u-\ln S(t)\right\},
\end{aligned}
$$

where

$$
\begin{gathered}
I_{S f}(t, \tau)=I_{S}(t, \tau)+I_{f}(t, \tau) \\
\sigma_{S f}^{2}(t, \tau)=\sigma_{S}^{2}(t, \tau)+\sigma_{f}^{2}(t, \tau)+2 \operatorname{cov}\left(I_{f}, I_{S}\right) \\
\operatorname{cov}\left(I_{f}, I_{S}\right)=\int_{t}^{\tau} d u \int_{0}^{\tau-u} \sigma(u, x) d x \int_{0}^{\bar{T}} b(u, y) \rho(x, y) d y .
\end{gathered}
$$

From (47)-(54), we have the following theorem.

Theorem 8. For the exogenous shock,

(1) if $J_{f}<0$, then $f_{C D} \uparrow$,

(2) if $J_{S}>0$, then

$$
\widetilde{P}\left(K_{\tau}<S(\tau)\right) \uparrow, \widetilde{P}(P(\tau) \geq S(\tau)) \downarrow, P(\tau)-S(\tau) \downarrow,
$$

where $f_{C D}$ is dependent on the total impact,

(3) if $J_{S}+J_{f}>0$, then

$$
(P(\tau)-S(\tau))^{+} \downarrow, \widetilde{P}(S(\tau)<P(\tau)) \downarrow, f_{C D} \downarrow,
$$

(4) if $J_{S}+J_{f}<0$, then

$$
(P(\tau)-S(\tau))^{+} \uparrow, \widetilde{P}(S(\tau)<P(\tau)) \uparrow, f_{C D} \uparrow .
$$

For the impact of covariation between $I_{S}$ and $I_{f}$, we have

$$
\begin{aligned}
& \text { (1) when } S(\tau)>K_{\tau}, \operatorname{cov}\left(I_{f}, I_{S}\right)>0 \text {, } \\
& \text { if } S(\tau) \uparrow \text {, then } f_{C D} \downarrow, \\
& \text { (2) when } S(\tau) \leq K_{\tau}, \operatorname{cov}\left(I_{f}, I_{S}\right)<0 \text {, } \\
& \text { if } S(\tau) \downarrow \text {, then } f_{C D} \uparrow .
\end{aligned}
$$

Remark 9. (1) The present default distance $\ln S(t)-\ln P(t)$ is negatively related with the default probability $P_{d}(S, P)$. Therefore, it negatively relates with the credit spread.

(2) The covariation between the economic basis and firm value will affect the default probability and the credit spread. If the economy is boom and the covariation is negative, then the credit spread will narrow along with the increasing firm 
value. If the economy is recession and the covariation is positive, then the credit spread would widen along with the decreasing firm value.

(3) The shocks cause jump in benchmark bond price and the firm value. When the exogenous shock increases the firm value, the default probability would decrease. Therefore, the embedded options and the credit spread tend to decrease. However, the call provision would offset this effect to some degree. The effect of exogenous shock depends on the total impact of $J_{f}$ and $J_{S}$. As Theorem 8 shows, if $J_{S}+J_{f}>0$, then the credit spread would narrow. If $J_{S}+J_{f}<0$, then the credit spread would widen.

(4) Due to the free boundary problems of American options and the firm's payout rate, we have not obtained the accurate pricing for the firm bond and the embedded American options.

\section{Conclusion}

We study the valuation of the firm bonds and their embedded options through an infinite dimensional jump-diffusion process. Some analytical results on corporate bond valuation, embedded options, and their exercise boundaries are obtained. By decomposing the corporate bond as benchmark bond minus or plus embedded options, analyzing the credit spread's effect factors, and dismantling the comprehensive effects of the exogenous shock on the embedded options, we find that the credit spread is affected by the firm value. Lower initial firm value implies higher credit spread. But the difference between the spreads is less than the difference of firm value times the risk-neutral default probability. A formal empirical test of the model's implications especially on the "credit spread puzzle" would be an interesting subject for future researches.

\section{Conflict of Interests}

The author declares that there is no conflict of interests regarding the publication of this paper.

\section{Acknowledgments}

Thanks are due to referees whose meaningful suggestions are very helpful to revise the paper. This work is supported by the Fundamental Research Funds for the Central Universities (JBK130401 and JBK131106).

\section{References}

[1] J. Amato and E. Remolona, "The credit spread puzzle," BIS Quarterly Review, pp. 51-63, 2003.

[2] E. J. Elton, M. J. Gruber, D. Agrawal, and C. Mann, "Explaining the rate spread on corporate bonds," Journal of Finance, vol. 56, no. 1, pp. 247-277, 2001.

[3] P. Collin-Dufresne, R. S. Goldstein, and J. S. Martin, “The determinants of credit spread changes," Journal of Finance, vol. 56, no. 6, pp. 2177-2207, 2001.

[4] K. J. M. Cremers, J. Driessen, and P. Maenhout, "Explaining the level of credit spreads: option-implied jump risk premia in a firm value model," Review of Financial Studies, vol. 21, no. 5, pp. 2209-2242, 2008.

[5] D. Duffie, J. Pan, and K. Singleton, "Transform analysis and asset pricing for affine jump-diffusions," Econometrica, vol. 68, no. 6, pp. 1343-1376, 2000.

[6] S. G. Kou and H. Wang, "Option pricing under a double exponential jump diffusion model," Management Science, vol. 50, no. 9, pp. 1178-1192, 2004.

[7] C. L. Yu, H. Li, and M. T. Wells, "Mcmc estimation of lévy jump models using stock and option prices," Mathematical Finance, vol. 21, no. 3, pp. 383-422, 2011.

[8] F. A. Longstaff, S. Mithal, and E. Neis, "Corporate yield spreads: default risk or liquidity? New evidence from the credit default swap market," Journal of Finance, vol. 60, no. 5, pp. 2213-2253, 2005.

[9] D. P. Kennedy, “The term structure of interest rates as a Gaussian random field," Mathematical Finance, vol. 4, no. 3, pp. 247-258, 1994.

[10] D. P. Kennedy, "Characterizing Gaussian models of the term structure of interest rates," Mathemnatical Finance, vol. 7, no. 2, pp. 107-118, 1997.

[11] R. S. Goldstein, "The term structure of interest rates as a random field," Review of Financial Studies, vol. 13, no. 2, pp. 365-384, 2000.

[12] P. Santa-Clara and D. Sornette, "The dynamics of the forward interest rate curve with stochastic string shocks," Review of Financial Studies, vol. 14, no. 1, pp. 149-185, 2001.

[13] R. C. Merton, "On the pricing of corporate debt: the risk structure of interest rates," Journal of Finance, vol. 29, no. 2, pp. 449470, 1974.

[14] F. Black and J. C. Cox, "Valuing corporate securities: some effects of bond indenture provisions," Journal of Finance, vol. 31, no. 2, pp. 351-367, 1976.

[15] M. J. Brennan and E. S. Schwartz, "Analyzing convertible bonds," Journal of Financial and Quantitative Analysis, vol. 15, no. 4, pp. 907-929, 1980.

[16] F. A. Longsta and E. S. Schwartz, "A simple approach to valuing risky fixed and oating rate debt," Journal of Finance, vol. 50, pp. 789-819, 1995.

[17] R. Geske, "The valuation of corporate liabilities as compound options," Journal of Financial and Quantitative Economics, vol. 12, no. 4, pp. 541-552, 1977.

[18] M. J. Brennan and E. S. Schwartz, "Convertible bonds: valuation and optimal strategies for call and conversion," Journal of Finance, vol. 32, no. 5, pp. 1699-1715, 1977.

[19] E. O. Fischer, R. Heinkel, and J. Zechner, "Dynamic capital structure choice: theory and tests," Journal of Finance, vol. 44, no. 1, pp. 19-40, 1989.

[20] H. Leland and K. Toft, "Optimal capital structure, endogenous bankruptcy, and the term structure of credit spreads," Journal of Finance, vol. 51, no. 3, pp. 987-1019, 1996.

[21] V. V. Acharya and J. N. Carpenter, "Corporate Bond Valuation and Hedging with Stochastic Interest Rates and Endogenous Bankruptcy," Review of Financial Studies, vol. 15, no. 5, pp. 13551383, 2002.

[22] S. E. Shreve, Stochastic Calculus for Finance II: Continuous-Time Models, Springer Finance, 2004.

[23] S. Fan, "A stochastic string with a compound Poisson process," Abstract and Applicated Analysis, vol. 2013, Article ID 857678, 8 pages, 2013. 


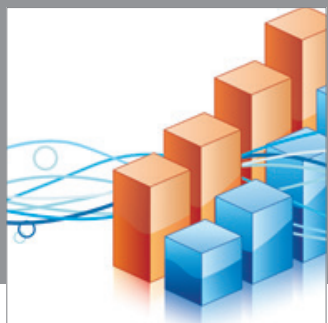

Advances in

Operations Research

mansans

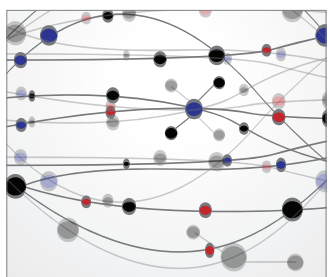

The Scientific World Journal
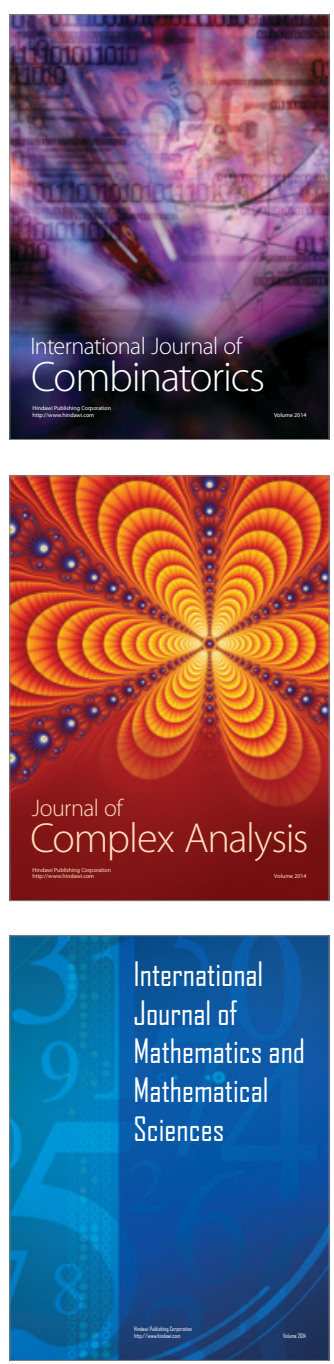
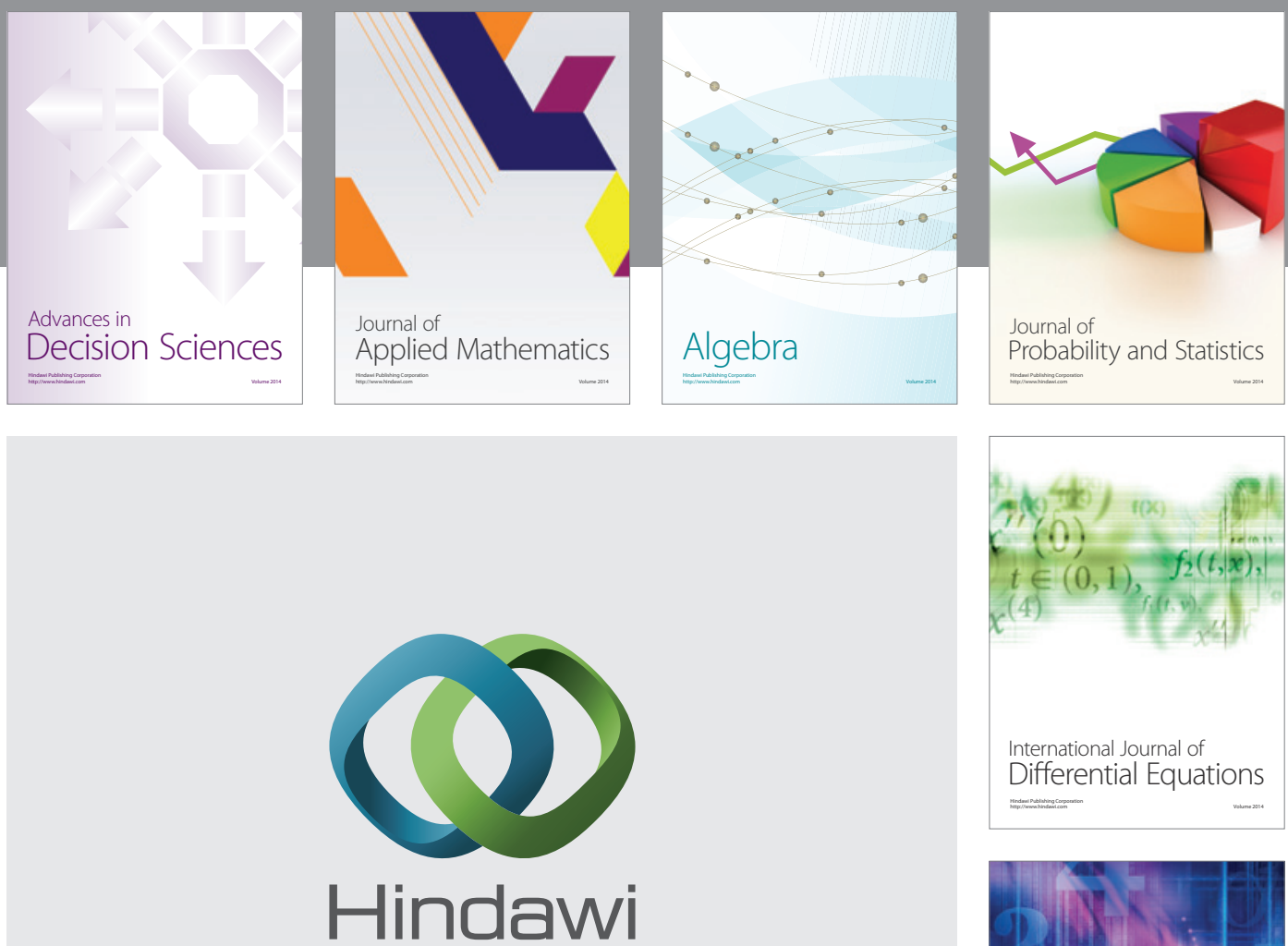

Submit your manuscripts at http://www.hindawi.com
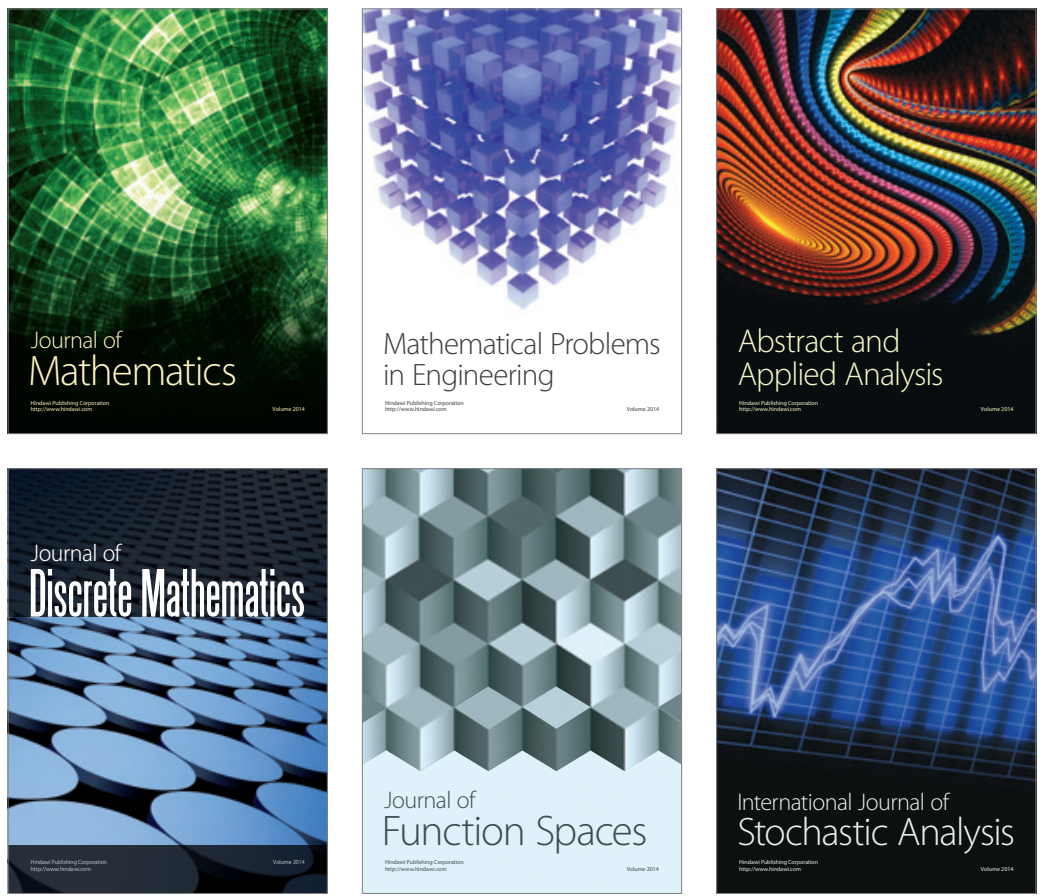

Journal of

Function Spaces

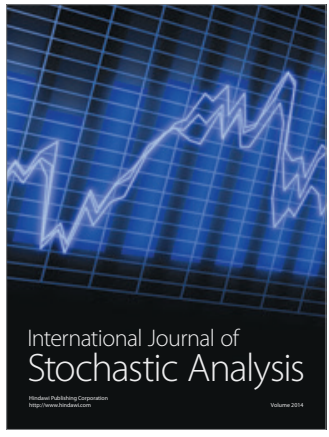

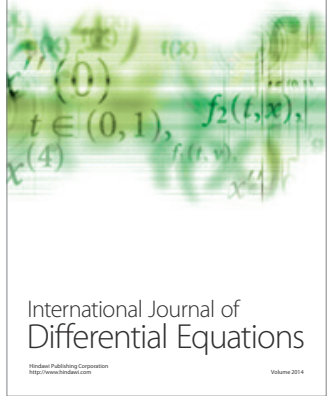
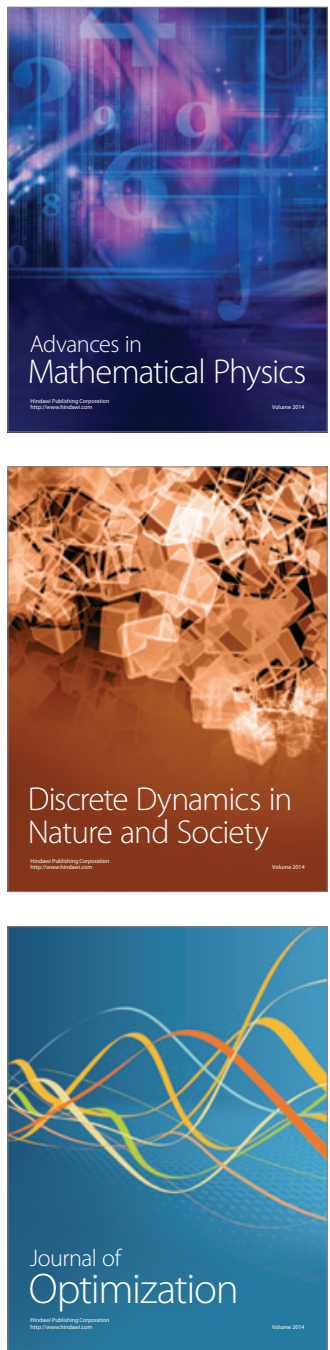